\title{
TITLE:
}

\section{TIGHT CONTROL OF BLOOD GLUCOSE WITH I.V. INSULIN: EFFICACY AND}

SAFETY.

\section{AUTHORS:}

Martin R. Lessard MD, Louis Bergeron M.Sc., Vivianne Bergeron MD, Anick Rioux M.Sc., 1 Geneviève Larouche M.Sc., Jocelyne Moisan PhD, François Lauzier MD, François LeBlanc MD

\section{AFFILIATION:}

Departments of Anesthesiology (Division of critical care), Medicine and Pharmacy, Université Laval and CHAUQ (Hô ptal Enfant-Jésus), $140118^{\mathrm{e}}$ rue, Québec, QC, G1J 1 Z4.

INTRODUCTION: Van den Berghe et al. have reported that tight control of blood glucose (BG) with intensive insulin therapy can reduce morbidity and mortality with a low incidence of side effects in critically ill patients. ${ }^{1}$ However, such a protocol may be difficult to implement outside of a research setting. This retrospective observational cohort study was designed to evaluate the efficacy and the safety of a continuous insulin infusion protocol (CIIP) in the usual ICU setting and to identify causes of hypoglycemia.

METHODS: The CIIP was run by the nursing staff and targeted a BG level between 4.5 and 8.0 mmol/L. BG was measured by capillary sampling every hour until 3 consecutive BG measurements were in the target range, then every 3 hours. Hypoglycemia and severe hypoglycemia were defined as $\mathrm{BG}$ level less than $3.5 \mathrm{mmol} / \mathrm{L}$ and $2.5 \mathrm{mmol} / \mathrm{L}$ respectively. Medical records of 98 consecutive ICU patients who received the CIIP were reviewed. Hypoglycemic events were further investigated to identify risk factors. Data are presented as mean \pm SD.

RESULTS: Patients were aged $58 \pm 18$ yr, APACHE II score was $23 \pm 8$, and BG level before starting the CIIP was $11.5 \pm 4.8 \mathrm{mmol} / \mathrm{L}$. CIIP was administered during $177 \pm 188 \mathrm{hr}$. Time between start of the CIIP and 3 consecutives BG levels within the target range was $16.9 \pm 19.6$ hr. Thereafter mean BG level during CIIP was $7.1 \pm 1.0 \mathrm{mmol} / \mathrm{L}$. Hypoglycemia and severe hypoglycemia occurred in 2.5 cases per 1000 patient-hr and 0.8 cases per 1000 patients-hr respectively. No complication of hypoglycemia was observed. Forty-two percent and $37 \%$ of the 19 severe hypoglycemic events were associated with corticosteroids given by intermittent IV boluses or with inappropriate application of the CIIP by the nursing staff, respectively.

CONCLUSIONS: Tight control of BG with a CIIP, outside of a research setting, is feasible and safe in the ICU. Corticosteroid use by intermittent boluses was associated with rebound hypoglycemia.

REFERENCES: 1. N Engl J Med 345: 1359-67 